\title{
Potential Genes and Mechanisms Linking Intracerebral Hemorrhage and Depression: A Bioinformatics-Based Study
}

This article was published in the following Dove Press journal: International Journal of General Medicine

\author{
Cuihua Zou ${ }^{1,2, *}$ \\ Xiaohua Huang (iD ${ }^{1,2, *}$ \\ Xuequn Lan' \\ Xiaorui Huang (iD) \\ Yun Feng' \\ Jianmin Huang' \\ Lanqing Meng' \\ Chongdong Jian' \\ Donghua Zou (iD) ${ }^{3}$ \\ Jie Wang ${ }^{4}$ \\ Xuebin $\mathrm{Li}^{1,2}$ \\ 'Department of Neurology, The Affiliated \\ Hospital of Youjiang Medical University \\ for Nationalities, Baise, Guangxi, 533000, \\ People's Republic of China; ${ }^{2}$ Department \\ of Neurology, Youjiang Medical University \\ for Nationalities, Baise, Guangxi, 533000, \\ People's Republic of China; ${ }^{3}$ Department \\ of Neurology, The Fifth Affiliated \\ Hospital of Guangxi Medical University, \\ Nanning, Guangxi, 530022, People's \\ Republic of China; ${ }^{4}$ Department of \\ Nephrology, The Affiliated Hospital of \\ Youjiang Medical University for \\ Nationalities, Baise, Guangxi, 533000 \\ People's Republic of China
}

*These authors contributed equally to this work

Correspondence: Jie Wang; Xuebin Li The Affiliated Hospital of Youjiang Medical University for Nationalities, 18 Zhongshan er Road, Baise, Guangxi, 533000, People's Republic of China Email yyfywj@I26.com; yyfylxb@I63. com
Purpose: The purpose of this study was to investigate the potential pathogenic mechanisms of post-intracerebral hemorrhage depression.

Methods: Profiles of gene expression in brain tissue of patients with intracerebral hemorrhage $(\mathrm{ICH})$ or depression were downloaded from the Gene Expression Omnibus (GEO) database. We analyzed differentially expressed genes (DEGs) for the two diseases separately. With these DEGs, we conducted an enrichment analysis based on Gene Ontology (GO) and Kyoto Encyclopedia of Genes and Genomes (KEGG) as well as cross-talk analysis, then we identified hub bridge genes using integrated bridge landscape analysis.

Results: We found 131 DEGs for interaction between ICH and depression. In the enrichment analysis, we found 55 GO terms and KEGG pathways involving interacting genes of ICH and depression, and $10 \mathrm{GO}$ terms and $10 \mathrm{KEGG}$ pathways most significantly related to cross-talk between ICH and depression. In the integrated bridge landscape analysis, we identified 20 hub bridge genes. In further analysis, we found that hub bridge genes $H L A-A, H M O X 1$, and JUN related to endocytosis, cell adhesion, and phagosomes may exert their effects through the dopamine (DA) system and the serotonergic pathway post-ICH depression. HLA-A may play a role in the occurrence and development of $\mathrm{ICH}$ and depression through immune mediation and cell adhesion. HMOXI and $J U N$ may participate in the mechanism by interacting with HLA-A.

Conclusion: Through bioinformatics analysis, we identified potential hub bridge genes and pathways related to post-ICH depression. Our study provides references for further research on mechanisms on the pathogenesis of post-ICH depression.

Keywords: intracerebral hemorrhage, depression, differentially expressed genes, hub bridge genes

\section{Introduction}

The global population is aging, and the prevalence and mortality of stroke are on the rise. According to the Global Burden of Disease Study 2016, the highest agestandardized incidences of stroke are observed in eastern Asia, especially in China, where incidence is 354 cases [95\% confidence interval (CI) 331-378] per 100,000 person-years. ${ }^{1}$ In particular, ischemic stroke and hemorrhagic stroke accounted for about $70 \%$ and $28 \%$ of all stroke cases in China, respectively. ${ }^{2}$

Many survivors of stroke suffer from mental and cognitive disorders. ${ }^{3}$ Poststroke depression (PSD), often occurring 3-6 months after stroke, is one of the most common mental disorders. ${ }^{4}$ PSD seriously affects the recovery of neurological 
function in stroke patients and increases the incidence of disability and mortality. ${ }^{5,6}$ The incidence of PSD in stroke survivors is $33 \% \%^{7}$ and the pathogenesis of PSD is complex. At present, research on the mechanisms of PSD has focused on depression after ischemic stroke and has neglected depression after hemorrhagic stroke.

Intracerebral hemorrhage $(\mathrm{ICH})$ is a serious type of stroke, and it is associated with the highest mortality among all stroke types. ${ }^{8}$ According to the Framingham Heart Study in the USA, the incidence of ICH was 43 cases per 100,000 person-years in the period 1948-2016. An age-stratified analysis indicated a continued increase in ICH incidence among patients 75 years and older, with incidence reaching 176 cases per 100,000 person-years in the period 2000-2016. ${ }^{9}$ The pathogenesis of ICH is not completely clear, although studies have implicated miR126, cerebral cavernous malformation (CCM) proteins, Ang-1/Tie2, Smad4, Notch3, lipocalin-2, and 1q22. ${ }^{10-17}$

Post-ICH depression is common, affecting around 20\% of ICH survivors, and it is associated with late exacerbation of $\mathrm{ICH}^{6,18}$ In addition, $\mathrm{ICH}$ is often accompanied by neurological dysfunction such as aphasia and related language disorders, and cognitive difficulties. These complications often mask depressive symptoms, making it difficult for physicians to diagnose depression and prescribe the appropriate treatment. Therefore, it is important to clarify the pathogenesis of post-ICH depression and find new prevention, diagnosis, and treatment methods. Studies have implicated inflammation, oxidative stress, apoptosis, and autophagy. ${ }^{19}$ However, the mechanisms of post-ICH depression are not fully understood.

There is a bi-directional relationship between stroke and depression, and some common mechanisms and risk genes between the two diseases have been identified. ${ }^{4}$ However, we are unaware of comprehensive, unbiased, integrated bioinformatics analysis of the mechanisms linking ICH and depression. In this study, we performed such an analysis to investigate the potential genes and mechanisms linking ICH and depression in order to provide a new theoretical basis for revealing the mechanisms behind the pathogenesis of post-ICH depression.

\section{Materials and Methods}

\section{Data Collection and Processing}

This study was based on Gene Expression Omnibus (GEO) datasets from post-mortem patients (www.ncbi. $\underline{\text { nlm.nih.gov/geo/). We included one dataset of } \mathrm{ICH} \text { and }}$ seven datasets of depression: GSE24265 [11 brain specimens of $\mathrm{ICH}$, including 4 perihematomal $(\mathrm{PH})$ areas, $\mathrm{PH}$ areas suspected to present edema identified by neuroradiology images from 4 deceased patients who had a supratentorial intracerebral hemorrhage, 4 contralateral gray (CG) matter, and 3 contralateral white (CW) matter], GSE87610 [76 brain specimens of dorsolateral prefrontal cortex (DLPFC) from patients with depression], GSE92538 [76 brain samples of DLPFC from patients with depression], GSE54562 [10 brain samples of anterior cingulate cortex (ACC) from patients with depression], GSE54572 [12 brain samples of ACC from patients with depression], GSE54565 [16 brain samples of ACC from patients with depression], GSE54564 [21 brain samples of amygdala (AMY) from patients with depression], and GSE24095 [15 brain specimens of the hippocampus dentate gyrus (DG) and 15 brain specimens of the hippocampus CA1 subregion from patients with depression]. The data are summarized in Table 1.

GSE54572 and GSE54565 datasets were based on the GPL570 Affymetrix Human Genome U133 Plus 2.0 Array platform (Affymetrix; Thermo Fisher Scientific, Waltham, MA, USA) and were combined to remove different batches. The GSE92538 dataset was based on the GPL10526 Affymetrix GeneChip Human Genome HGU133 Plus 2 Array platform (Affymetrix; Thermo Fisher Scientific), and the GPL17027 dataset was based on the Affymetrix Human Genome U133A Array platform (Affymetrix; Thermo Fisher Scientific). These datasets were divided into batches. We used the "normalizeBetweenArrays" function of the limma package $^{20}$ in $\mathrm{R}$ to standardize the data. If a gene had two or more expression values, we used the average as the gene expression value.

\section{Principal Component Analysis and Screening for Differentially Expressed Genes}

Principal component analysis (PCA) was performed using the FactoMineR (https://www.r-project.org/) and factoextra (https://github.com/cran/factoextra) packages in $\mathrm{R}$ to evaluate the data in each dataset.

The data were preprocessed and then screened to identify differentially expressed genes (DEGs) using the unpaired $t$-test provided by the limma package in $\mathrm{R}$. The threshold of significance was defined as $\mathrm{P}<0.01$ in the dataset of ICH. Gene expression patterns were compared 
Table I Data Collection

\begin{tabular}{|l|l|l|c|}
\hline Condition & Tissue & GEO Datasets & $\begin{array}{c}\text { Number of Brain } \\
\text { Samples }\end{array}$ \\
\hline Depression & DLPFC & GSE87610 & 76 \\
& & GSE92538- & 76 \\
& & GSL10526 & GPL17027 \\
\hline Depression & ACC & GSE54562 & 76 \\
\hline Depression & AMY & GSE54564 & 10 \\
\hline Depression & CAI & GSE24095 & 12 \\
\hline Depression & DG & GSE24095 & 21 \\
\hline ICH & PH & GSE24265 & 15 \\
\hline
\end{tabular}

Abbreviations: ACC, anterior cingulate cortex; AMY, amygdala; CAI, CAI subregions of hippocampus; DG, dentate gyrus of hippocampus; DLPFC, dorsolateral prefrontal cortex; ICH, intracerebral hemorrhage; PH, perihematomal.

between PH and contralateral healthy tissue from the same individuals. To exclude inherent differences between white matter and grey matter not influenced by ICH, both white and grey matter from the contralateral hemisphere were independently compared against the $\mathrm{PH}$ tissue, then white and grey matter from the contralateral hemisphere were compared with each other. Therefore, three comparisons of gene expression were calculated per individual: $\mathrm{PH}$ vs CG, $\mathrm{PH}$ vs $\mathrm{CW}$, and $\mathrm{CW}$ vs CG. DEGs in ICH were defined as intersection genes for the comparisons $\mathrm{PH}$ vs $\mathrm{CG}$ and $\mathrm{PH}$ vs $\mathrm{CW}$ that showed consistent expression, minus the DEGs from the comparison CG vs CW.

For the depression DEGs, we used the same tissue in the dataset and selected the DEGs that showed consistent up- or down-regulation and that fell within the top $100 \mathrm{Pi}$ values, where $\left[\mathrm{Pi}=-\lg (\mathrm{p})^{*}|\log \mathrm{FC}|\right], \mathrm{p}$ is $\mathrm{p}$-value and $\mathrm{FC}$ is fold chang. Values for genes present in multiple datasets were defined as the sum of the values across the datasets. Two-way hierarchical clustering was performed on the DEG profiles of ICH based on Euclidean distance using pheatmap (https://github.com/cran/pheatmap).

\section{Analysis of Functional Enrichment}

We used the clusterProfiler ${ }^{21}$ package to examine DEGs of ICH and depression based on enrichment of Gene Ontology (GO) functions and Kyoto Encyclopedia of Genes and Genomes (KEGG) pathways. We screened biological process GO terms and KEGG pathways that might be related to $\mathrm{ICH}$ and depression using Gene Set Enrichment Analysis (GSEA) (GSEA2-2.2.4, Java version). ${ }^{22}$ The datasets c5.bp.v6.2.symbols.gmt and c2. cp.kegg.v6.2.symbols.gmt in the MsigDB V6.2 database $^{23}$ were used as reference gene sets, and GSEA was performed according to default parameters. We set nominal $\mathrm{P}<0.05$ as the threshold for significant enrichment.

\section{Cross-Talk Analysis}

DEGs of ICH and depression were divided into six modules: the ICH DEGs were one module, while the DEGs of the five tissues for depression were five modules. Based on the STRING database (version 11.0, https://string-db.org/) ${ }^{24}$, 131 interactions of ICH and depression were extracted from the cross-talk analysis. In the STRING database, the combined score was computed by combining the probabilities from the different evidence channels and corrected for the probability of randomly observing an interaction. The combined score of human proteins ranked 150 to 999 in the database. Only the interactions with combined scores $>700$ were included in the present study. The Sankey (https:// github.com/cran/sankey) diagram was used to demonstrate the functions and pathways of the interacting genes involved in ICH and depression.

\section{Integrated Bridge Landscape Network and Hub Bridge Genes}

Based on the cross-talk analysis, the bridge genes and their interaction pairs were analyzed in Cytoscape. ${ }^{25}$ The integrated bridge driving force was calculated as $\mathrm{W}=\mathrm{F} * \mathrm{Pi}$ *degree, where $\mathrm{F}$ is the driving force of bridge genes and degree represents the connectivity in the cross-talk network. The top 20 genes with the highest $\mathrm{W}$ values were defined as hub bridge genes. The GOSemSim ${ }^{26}$ package was used to calculate the driving force $\mathrm{F}$ of the function of the bridge genes, and the expression of bridge genes was explored, where expression was defined as the $\log _{2}$ value of the standardized expression.

\section{Results}

\section{Principal Component Analysis}

The flowchart of this study is shown in Figure 1. The results of the PCA showed that the first and second principal components (PCs) explained 13.2\% (GSE87610)$67.4 \%$ (GSE24265) of the variance in different datasets 


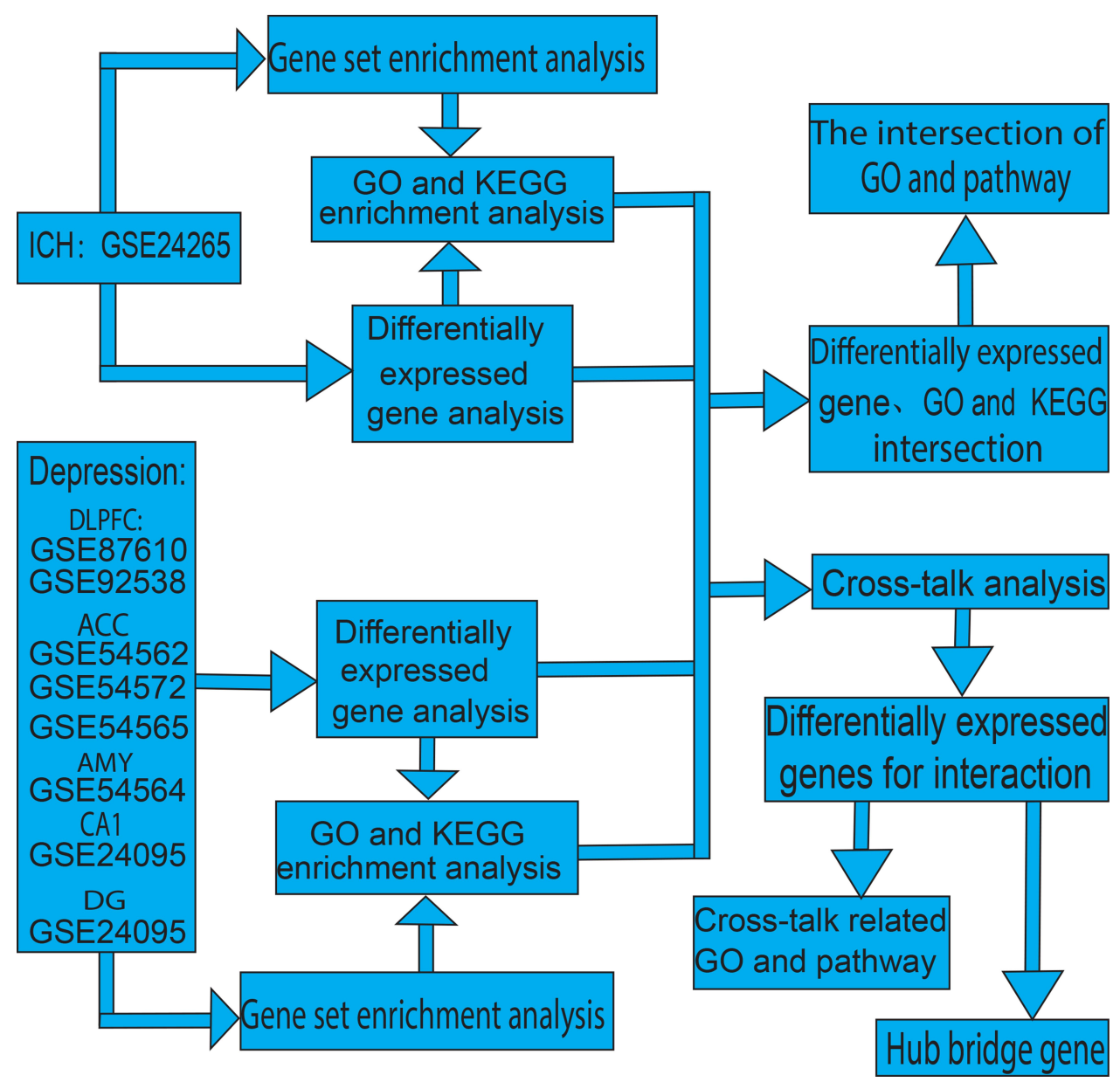

Figure I Study flowchart.

Abbreviations: ACC, anterior cingulate cortex; AMY, amygdala; CAI, hippocampus CAI subregion; DG, hippocampus dentate gyrus; DLPFC, dorsolateral prefrontal cortex; ICH, intracerebral hemorrhage.
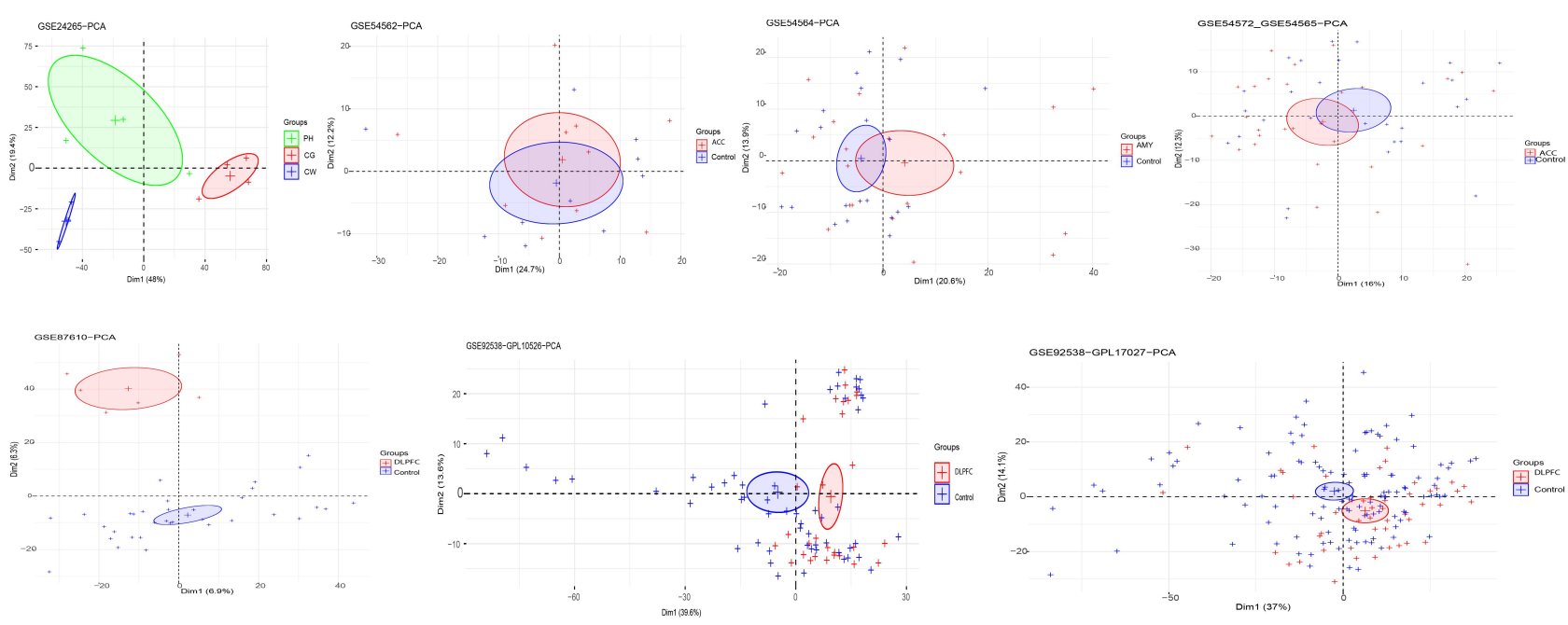

Figure 2 Principal component analysis (PCA) of the Gene Expression Omnibus (GEO) datasets included in the study.

Abbreviations: ACC, anterior cingulate cortex; AMY, amygdala; CW, contralateral white; CG, contralateral grey; DLPFC, dorsolateral prefrontal cortex; PH, perihematomal tissue. 
(Figure 2). Although the variance explained by PC1-2 depended on the dataset, the gene expression pattern was able to distinguish ICH and depression patients from normal control samples. Therefore, these datasets were used to analyze and identify links between ICH and depression. However, there was a greater degree of admixture in some PCs, which indicated the need to perform feature selection rather than use all genes.

\section{DEGs in ICH and Depression}

We identified DEGs for ICH and depression in comparison with healthy controls. For depression, we found 64 upregulated and 19 down-regulated genes in DLPFC samples (from GSE87610, GSE92538-GPL10526, and GSE92538GPL17027 datasets), 27 up-regulated and 73 downregulated genes in ACC samples (GSE54562 and GSE54572-GSE54565 datasets), 51 up-regulated and 49 down-regulated genes in AMY samples (GSE54564 dataset), 23 up-regulated and 77 down-regulated genes in CA1 subregions of the hippocampus (GSE24095 dataset), and 40 up-regulated and 60 down-regulated genes in the DG of the hippocampus (GSE24095 dataset) (Figure 3A). The DEGs of ICH and depression are summarized in Table 2.

We identified 113 DEGs in the $\mathrm{PH}$ tissue of ICH patients in the GSE24265 dataset, of which 82 genes were up-regulated and 31 were down-regulated (Figure 3B). We explored DEGs with consistently up-regulated and down-regulated expression in ACC and DLPFC tissue samples from patients with depression (Figure 3C). Cluster analysis showed that the expression patterns of the 113 DEGs were able to distinguish ICH from healthy control samples (Figure 3D).

\section{GO Terms and KEGG Pathways Related to Post-ICH Depression}

GO and KEGG enrichment analysis of DEGs in ICH and depression suggested that mechanisms of depression may be related to extracellular matrix (ECM)-receptor interactions and regulation of actin cytoskeleton (Figure 4A). ICH mechanisms appeared to be related to cell adhesion molecules (CAMs), endocytosis, and type 1 diabetes mellitus (Figure 4B). The enrichment analysis results were verified through GSEA. Figure $4 \mathrm{C}$ and Table S1 show 55 GO terms and KEGG pathways involving interacting genes of $\mathrm{ICH}$ and depression. The $55 \mathrm{GO}$ terms and KEGG pathways were mainly related to inflammation, immunity, stress, apoptosis, and autophagy. These GO terms and KEGG pathways may be involved in the pathogenesis of post-ICH depression.

\section{Cross-Talk Analysis}

In order to find more genes involved in mechanistic links between ICH and depression, we divided the five parts of depressed brain tissues (DLPFC, ACC, AMY, CA1, DG) and ICH DEGs into six modules (one module for ICH, five modules for depression) and conducted cross-talk analysis. We identified 131 DEGs for the interaction (Figure 5A). DEGs for interaction in ICH and depression were considered to bridge the two conditions. DEGs for interaction corresponding to diseases and tissues were defined as bridge genes, and the interaction between molecules encoded by those genes was defined as a bridge interaction.

\section{GO Terms and KEGG Pathways Related to Cross-Talk Genes}

Figure 5B shows the most significant GO terms related to cross-talk genes between ICH and depression. These terms included response to hypoxia, response to nutrient levels, negative regulation of DNA binding, positive regulation of neurological system processes, neuronal death, synapse organization, autophagy, cellular iron ion homeostasis, intrinsic apoptotic signaling pathway, and regulation of apoptotic signaling pathways. The most significant KEGG pathways related to cross-talk genes between ICH and depression included CAMs, type I diabetes mellitus, cellular senescence, regulation of actin cytoskeleton, endocytosis, ECM-receptor interaction, phagosome, neurotrophin signaling pathway, ferroptosis, and the Toll-like receptor signaling pathway (Figure 5C).

Supplementary tables show the GO terms (Tables S2-

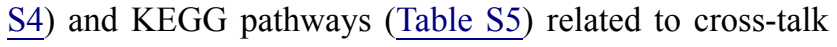
genes.

\section{Integrated Bridge Landscape Network and Hub Bridge Genes}

Using bridge genes, the pathways in which bridge genes are involved and other related genes in the pathway were used to build a network, which was considered the post-ICH depression-related gene regulatory network (Figure 5D). Any of these bridge genes may interact with other genes in the network to regulate relevant functional pathways, and thus, may play a role in the pathogenesis of post-ICH depression. We integrated the network to compute the function driving 


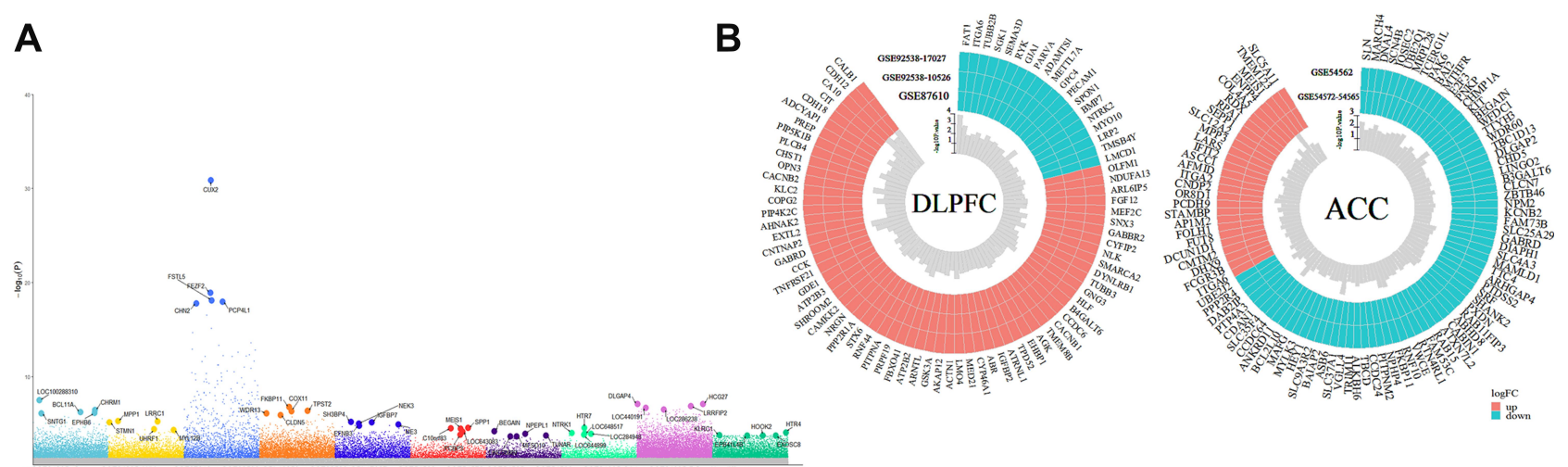

C
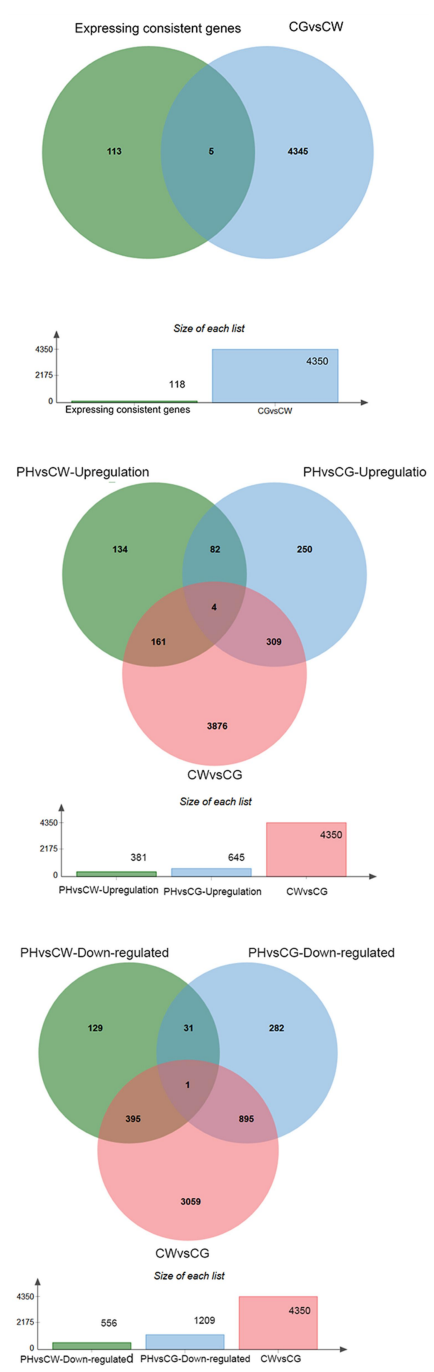

D

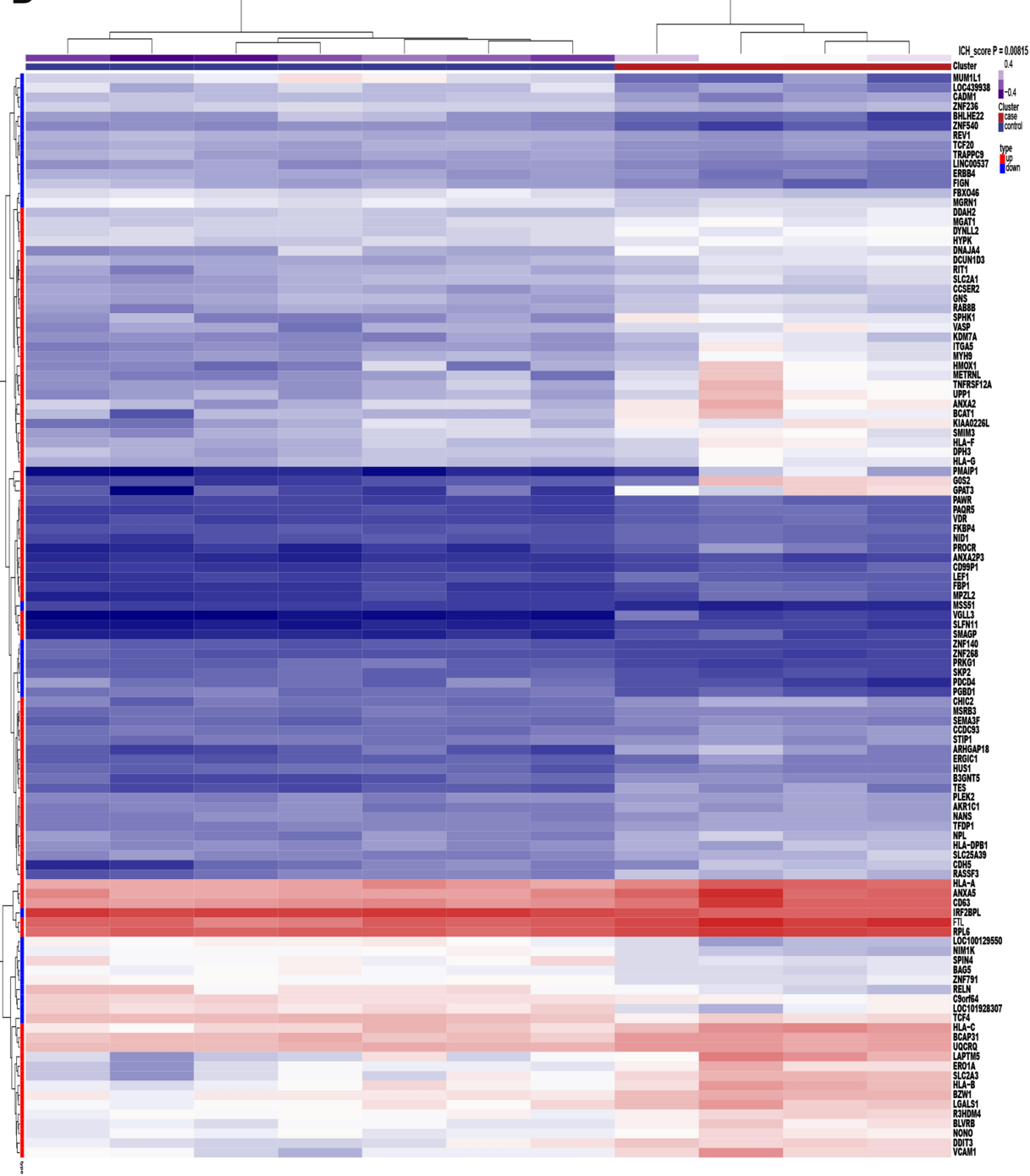

Figure 3 Analysis of differentially expressed genes (DEGs) and their clustering. (A) DEGs of intracerebral hemorrhage (ICH) and depression tissue samples. The genes with the most significant expression differences in each dataset are labeled. (B) DEGs of ICH. (C) DEGs of ACC and DLPFC tissues from patients with depression. (D) Cluster analysis heatmap showing how the expression patterns of these DEGs can distinguish ICH from normal control tissues.

Abbreviations: ACC, anterior cingulate cortex; CG contralateral grey; CW, contralateral white; DLPFC, dorsolateral prefrontal cortex; PH, perihematomal tissue.

force F (Figure 6) using the gene expression Pi value (taking the mean value across multiple datasets), defined as $\mathrm{Pi}=-\mathrm{lg}$ $(\mathrm{p}) *|\log 2 \mathrm{FC}|$ (Figure $7 \mathrm{~A}$ ). The integrated bridge driving force $\mathrm{W}$ was $\mathrm{W}=\mathrm{F} * \mathrm{Pi}$ * degree (where "degree" means the degree of connectivity in the cross-talk network). The $\mathrm{W}$ values of bridge genes are reported in Table S6. The 
Table 2 DEGs in ICH and Depression

\begin{tabular}{|c|c|c|c|c|}
\hline \multirow[t]{2}{*}{ Condition } & \multirow[t]{2}{*}{ Tissue } & \multirow{2}{*}{$\begin{array}{l}\text { GEO } \\
\text { Datasets }\end{array}$} & \multicolumn{2}{|c|}{ Number of DEGs } \\
\hline & & & $\begin{array}{c}\text { Up- } \\
\text { Regulated }\end{array}$ & $\begin{array}{l}\text { Down- } \\
\text { Regulated }\end{array}$ \\
\hline Depression & DLPFC & $\begin{array}{l}\text { GSE87610, } \\
\text { GSE92538- } \\
\text { GPLI0526, } \\
\text { GSE92538- } \\
\text { GPLI } 7027\end{array}$ & 64 & 19 \\
\hline Depression & $\mathrm{ACC}$ & $\begin{array}{l}\text { GSE54562, } \\
\text { GSE54572, } \\
\text { GSE54565 }\end{array}$ & 27 & 73 \\
\hline Depression & AMY & GSE54564 & 51 & 49 \\
\hline Depression & CAI & GSE24095 & 23 & 77 \\
\hline Depression & DG & GSE24095 & 40 & 60 \\
\hline $\mathrm{ICH}$ & $\mathrm{PH}$ & GSE24265 & 82 & 31 \\
\hline
\end{tabular}

Abbreviations: ACC, anterior cingulate cortex; AMY, amygdala; CAI, CAI subregions of hippocampus; DEGs, differentially expressed genes; DG, dentate gyrus of hippocampus; DLPFC, dorsolateral prefrontal cortex; ICH, intracerebral hemorrhage; $\mathrm{PH}$, perihematomal.

larger the $\mathrm{W}$ value, the more critical the nodes in the network. The top 20 gene nodes with the highest $\mathrm{W}$ values in the network, defined as hub bridge genes, were $A C T L 7 A$, RACGAP1, PRKAG1, CDH12, DYNLL2, VCAM1, SPHK1, GOLGA2, METRNL, KLRC1, JUN, ITGA2B, CDH5, TOLLIP, ANXA2, HLA-B, TAP2, ITGA5, HLA-A, and HMOX1 (Figure 7B and Table S6).

\section{Discussion}

Post-ICH depression is a common complication of ICH that significantly affects prognosis. Thus, it is crucial to clarify the mechanisms of post-ICH depression for its prevention and management. In this study, we aimed to investigate hub bridge genes and potential pathways of post-ICH depression based on the interaction network between ICH and depression. We identified hub bridge genes of post-ICH depression from the analysis of DEGs for interaction in $\mathrm{ICH}$ and depression. We found $55 \mathrm{GO}$ terms and KEGG pathways involving interacting genes of $\mathrm{ICH}$ and depression. In the GO and KEGG enrichment analysis of ICH and depression DEGs, we identified GO terms and pathways the most significant related to cross-talk. These hub bridge genes, functions and pathways may be related to post-ICH depression.

Previous studies found that activation of microglia after ICH leads to the production of proinflammatory cytokines, neuronal death, and structural deterioration. ${ }^{27}$ Activation of microglia and expression of proinflammatory cytokines are closely related to the pathogenesis of depression. $^{28}$ Inflammatory cytokines such as hs-CRP, TNF- $\alpha$, IL-1 $\beta$, IL-6, IL-18, and IFN- $\gamma$ reduce 5-hydroxytryptamine (5-HT), dopamine (DA), and norepinephrine signaling. ${ }^{29}$ The increased expression of inflammatory factors over-activates the hypothalamic-pituitary-adrenal (HPA) axis. ${ }^{30}$ The development of depression is closely related to imbalance of the HPA axis as well as signaling by 5 -HT, DA, and norepinephrine. ${ }^{31,32}$

In addition, after secondary brain injury in $\mathrm{ICH}$, many signaling pathways are activated, such as those related to inflammation, oxidative stress, autophagy and apoptosis. In particular, inflammation is related mainly to Toll-like receptors and signaling pathways dependent on NF-kB or PPAR- $\gamma$. Oxidative stress is related to nuclear factor erythroid-2 related factor 2 , as well as the PI3K/Akt and MAPK/p38 pathways. Autophagy is related to the mTOR signaling cascade and the NF-kB-mediated signaling pathway, while apoptosis is related to the death receptormediated apoptosis pathway, caspase-independent pathways, and mitochondrial apoptosis pathway. Moreover, oxidative stress, neuroinflammation, apoptosis and autophagy interact, and these pathways can serve as a bridge between ICH and depression. ${ }^{19}$ The 55 GO terms and KEGG pathways involving interacting genes of $\mathrm{ICH}$ and depression, which obtained in the analysis also involved immunity, apoptosis, stress, autophagy, and inflammation. These results align with previous studies.

In our work, we identified $A C T L 7 A, R A C G A P 1$, PRKAG1, CDH12, DYNLL2, VCAM1, SPHK1, GOLGA2, METRNL, KLRC1, JUN, ITGA2B, CDH5, TOLLIP, $A N X A 2$, HLA-B, TAP2, ITGA5, HLA-A, and HMOX1 as hub bridge genes between ICH and depression. The proteins encoded by these genes may be potential biomarkers for post-ICH depression.

Some of the hub bridge genes identified here have already been implicated in the pathophysiology of both ICH and depression. For example, AHNAK/p11/ANXA2 controls L-type voltage-gated calcium channels on the cell surface, which regulate neuronal activity induced by calcium signals and control depressive behaviors. ${ }^{33}$ In animal models, high levels of anti-ANXA2-antibodies can induce the accumulation of immunoglobulin $\mathrm{G}$ in the brain, which exerts an antidepressant effect. ${ }^{34}$ Polymorphisms of the $A N X A 2$ gene are associated with stroke, and the encoded protein, annexin A2, is involved in the regulated 
A

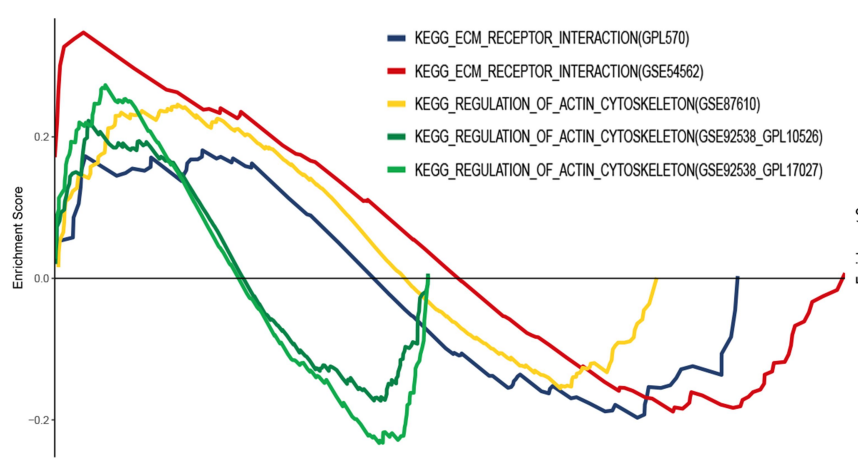

B

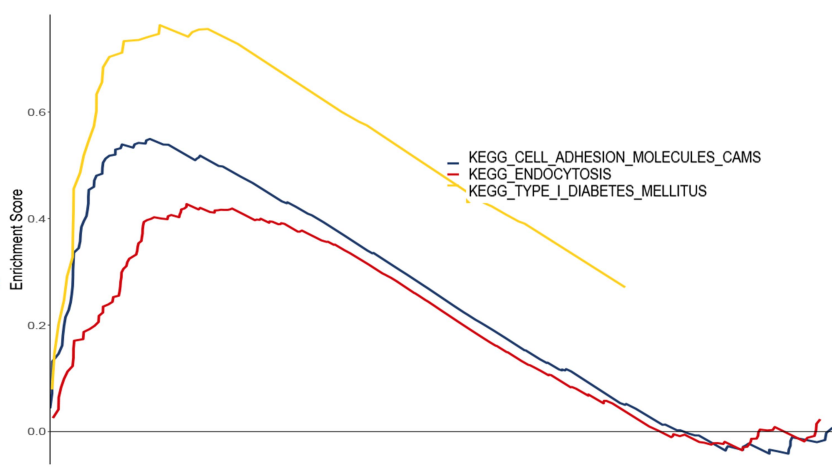

C

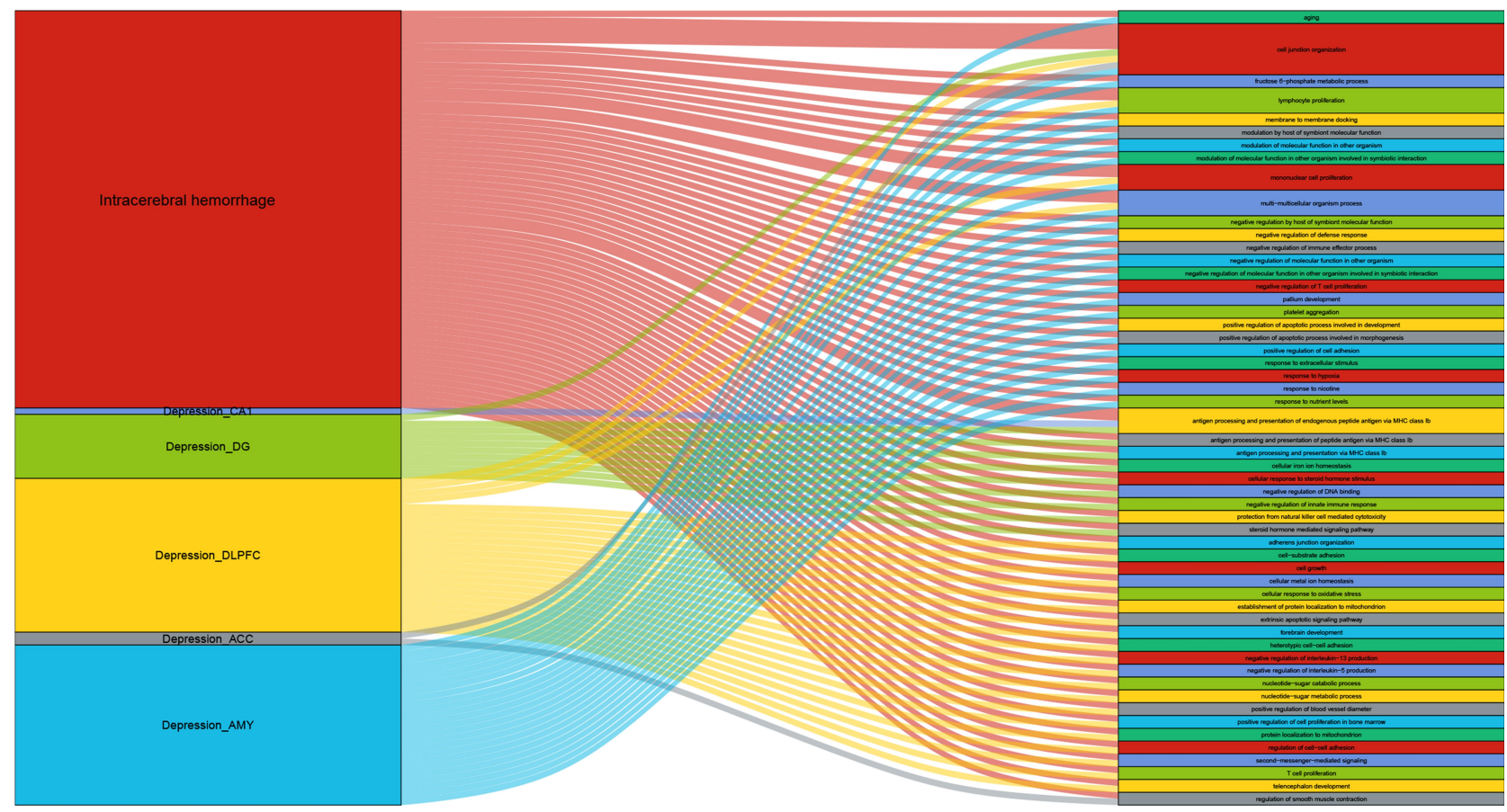

Figure 4 Gene Set Enrichment Analysis (GSEA) and the GO terms and KEGG pathways involving interacting genes of ICH and depression. (A) Kyoto Encyclopedia of Genes and Genomes (KEGG) pathways with the most significant enrichment in depression. (B) KEGG pathways with the most significant enrichment in ICH. (C) The GO terms and KEGG pathways involving interacting genes of $\mathrm{ICH}$ and depression.

generation of plasmin, the primary fibrinolytic protease regulating hemostasis and thrombosis. ${ }^{35}$

Several

HLA

haplotypes

(such $\mathrm{A} * 01 \sim \mathrm{B} * 52 \sim \mathrm{DRB} 1 * 15$, $\mathrm{A} * 24 \sim \mathrm{B} * 07 \sim \mathrm{DRB} 1 * 11$, $A * 24 \sim B * 35 \sim D R B 1 * 11$ and $A * 02 \sim B * 51 \sim D R B 1 * 11$ )
have been associated with an increased risk of stroke, while others (such as $A^{*} 26 \sim \mathrm{B}^{*} 38 \sim \mathrm{DRB} 1 * 11$ and $A * 03 \sim B * 35 \sim D R B 1 * 04)$ have shown protective effects against stroke. HLA-B $* 35, \mathrm{~b} * 52$ may affect stroke risk by changing the function of arterial endothelial cells. ${ }^{36}$ Meanwhile, the HLA system is related to the risk of bipolar manic-depressive illness: HLA-B*08:01 may help protect against it $^{37}$ and antidepressant treatment has been associated with altered levels of HLA antigens A and B in patients with this disorder. ${ }^{38}$

Antidepressants such as imipramine and fluoxetine can induce the expression of genes related to neuroprotection, such as heme oxygenase-1 (HMOXI, HO-1). ${ }^{39}$ At the same time, heme-binding protein promotes the migration and differentiation of endothelial progenitor cells through the HMOX1 pathway, and promotes the formation of new blood vessels, thus protecting the integrity of the bloodbrain barrier, synaptic plasticity, and neural function. ${ }^{40} \mathrm{In}$ addition, the presence of hemoglobin at the focus of ICH aggravates brain injury. Binding globin binds to hemoglobin, prevents its oxidation, and participates in 


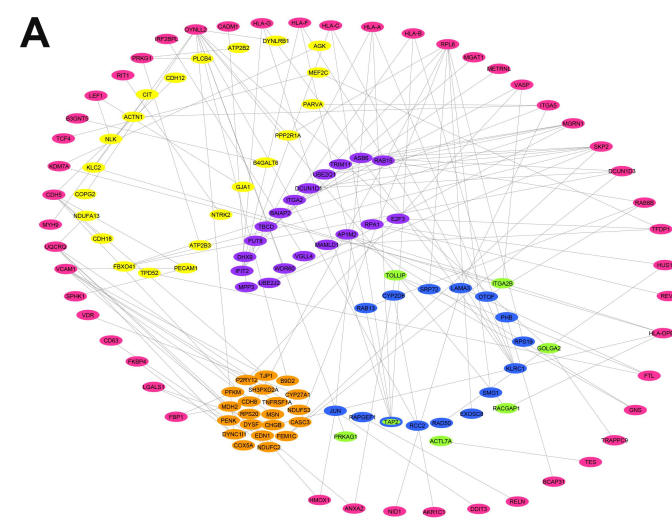

D

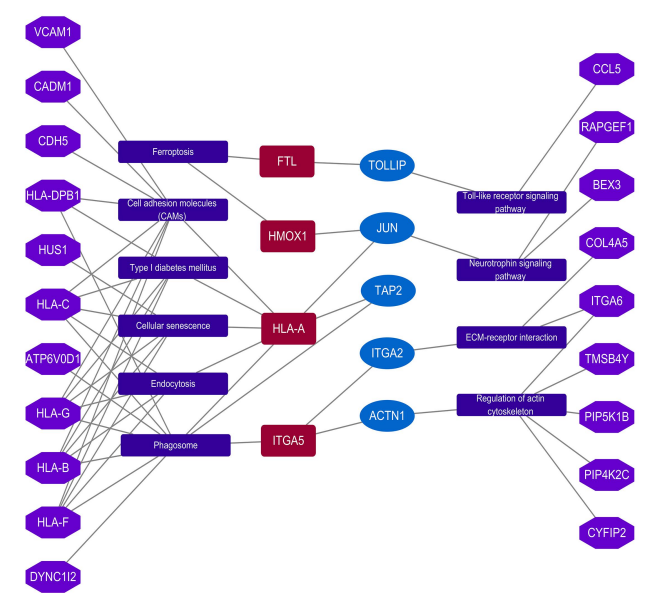

B

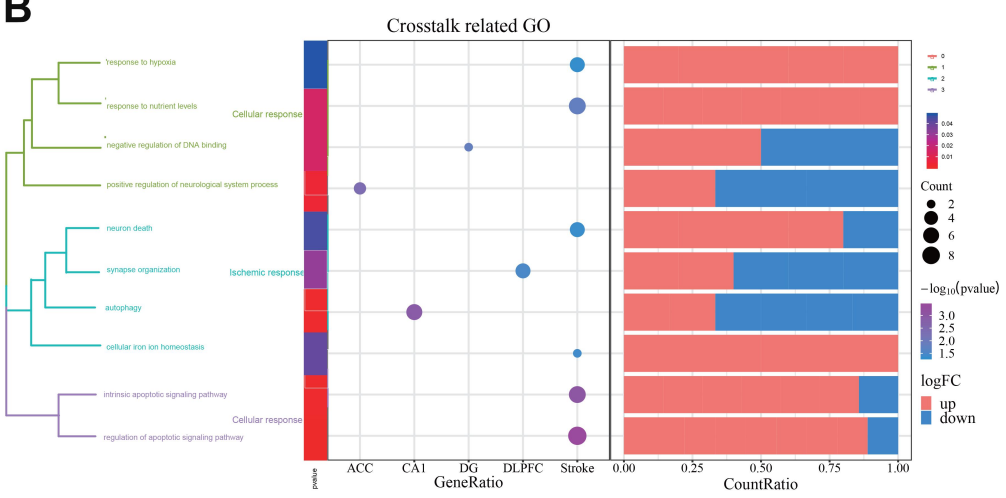

C

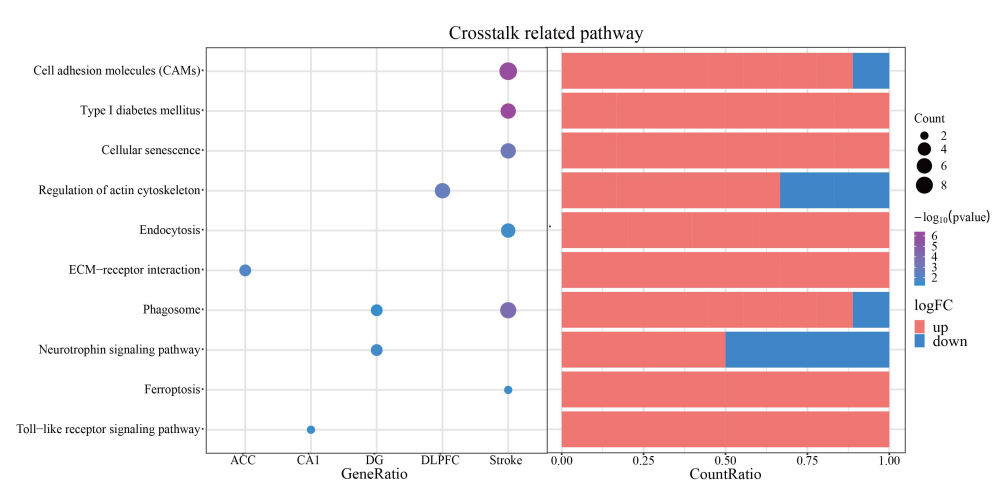

Figure 5 Cross-talk network, Gene Ontology (GO) terms and Kyoto Encyclopedia of Genes and Genomes (KEGG) pathways related to cross-talk and the integrated bridge landscape network. (A) Network of genes involved in cross-talk between ICH and depression. Pink indicates ICH-associated genes, while the other colors indicate depression-associated genes in the ACC (purple), AMY (orange), CAI (green), DG (blue), or DLPFC (yellow). TAP2 is the common gene of the hippocampal CAI subregion and DG. (B) GO terms and (C) KEGG pathways most significantly related to the cross-talk. The enrichment increases from blue to red. The larger the circle, the more significant the proportion of module genes present among GO functional entry genes or KEGG pathway entry genes. (D) The integrated bridge landscape network, including bridge genes, bridge genes related pathways, and other related genes in the pathway. Dark red indicates the bridge genes associated with ICH; blue, the bridge genes associated with depression. Bluish violet indicates the KEGG pathway, while purple indicates the other genes associated with KEGG.

Abbreviations: ACC, anterior cingulate cortex; CAI, hippocampus CAI subregion; DG, hippocampus dentate gyrus; DLPFC, dorsolateral prefrontal cortex; ICH, intracerebral hemorrhage.

neurotoxicity. HMOX1 is involved in this process. ${ }^{41}$ In experimental models of spontaneous $\mathrm{ICH}$, overexpression of HMOX1 in astrocytes after acute ICH mitigates the breakdown of the blood-brain barrier and short-term neurological deficits, exerts a neuroprotective effect, and improves outcomes. ${ }^{42}$

C-Jun N-terminal kinase (JNK) is a key mitogenactivated protein kinase $^{43}$ and an important regulator of inflammation and stress responses, and its expression is closely related to the development of central nervous system diseases. ${ }^{44}$ In ICH, levels of phosphorylated JNK 3 and C-Jun are up-regulated, and JNK contributes to neuronal apoptosis. ${ }^{45} \mathrm{JNK}$ also helps regulate the expression of pro-inflammatory cytokines, the phosphorylation of glucocorticoid receptors, and neuroinflammation-induced depression. $^{44}$
Previous studies support the idea that our hub bridge genes may be involved in the physiological and pathological processes of both ICH and depression, making them likely drivers of post-ICH depression. In the cross-talk analysis, $J U N$, the hub bridge gene of depression, interacts with $H L A-A$ and $H M O X 1$, the hub bridge genes of ICH. Previous studies have shown that HMOX1, JUN, and HLA$A$ are involved in the pathological processes of both ICH and depression. In our analysis, we found that the three interacting genes $H L A-A, H M O X 1$, and $J U N$ may play a role in the mechanism of ICH and depression by regulating the pathways of cellular senescence, endocytosis, type I diabetes mellitus, CAMs, phagosome, ferroptosis, and neurotrophin signaling.

In further analysis, we found that $H L A-A$ may interact with the other hub bridge genes, HLA-B, HLA-C, HLA-F, 

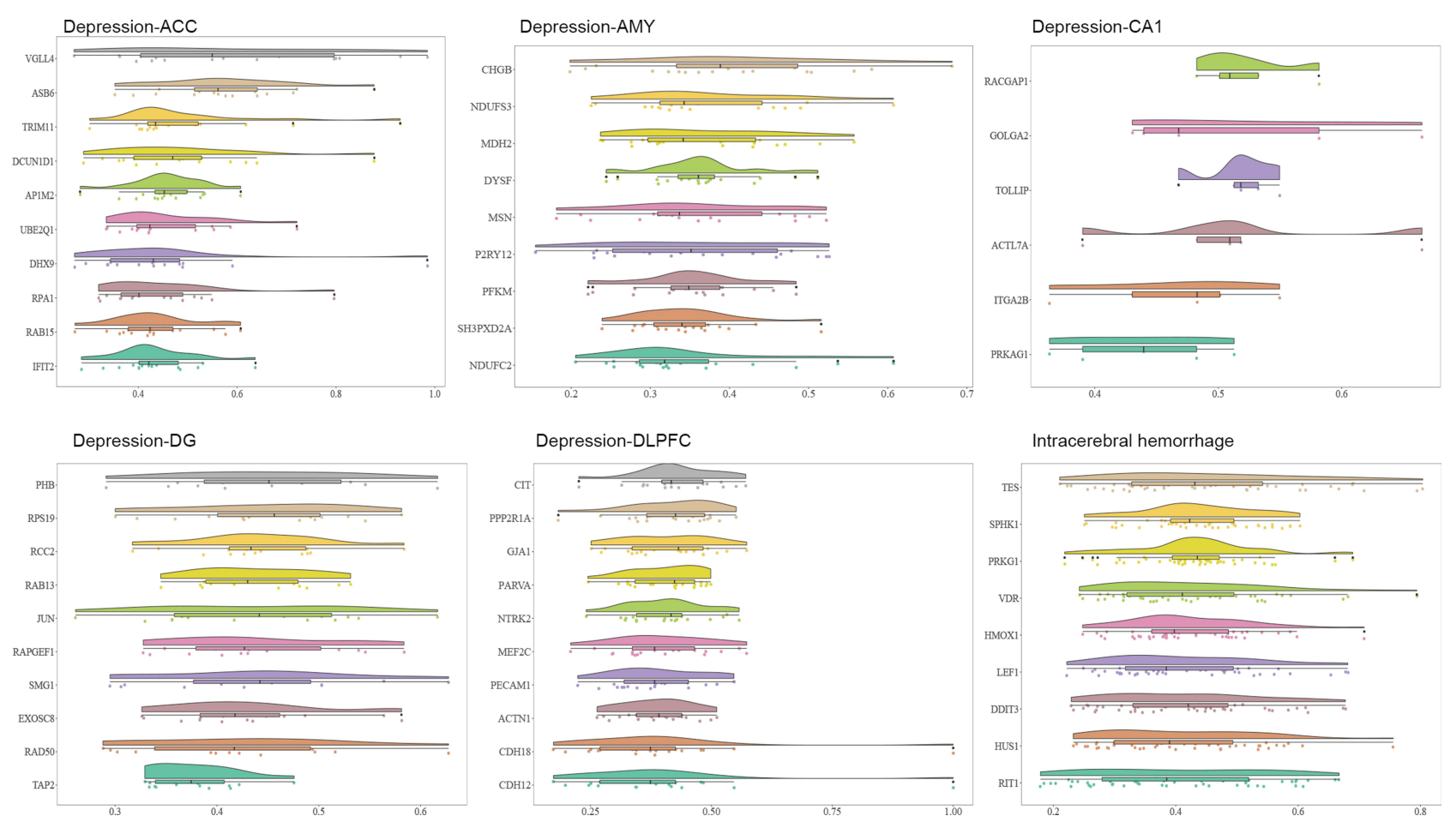

Figure 6 Results of functional driving forces of the bridge genes. Histograms of the genes with the greatest functional drive in each tissue. The $x$-axis represents a standardized score of gene functional driving force.

and $H L A-G$. These genes are involved in the regulation of endocytosis, CAMs, and phagosome pathways. This, together with the abnormal expression of other hub bridge genes in the pathway, such as HLA-DPB1, CDH5, $V C A M 1$, and $C A D M 1$, may play a role in immune mediation and cell adhesion, and therefore, in the occurrence and development of ICH and depression (Figure 8).

Lesions in the DA system play a key role in the pathophysiology of depression. Reduction in the number of DA neurons is associated with the severity of depression. Epothilone B can protect DA neurons by enhancing microtubule stability, mitigating damage to DA neurons and reducing the depressive behavior of post-ICH depressive patients. Protecting DA neurons can help reduce risk of post-ICH depression. ${ }^{46}$ The migration of mesencephalic dopaminergic neurons from the subventricular zone to their final positions in the substantia nigra compacta, ventral tegmental area, and retrorubral field is controlled by signaling from neurotrophic factors, CAMs, and extracellular matrix molecules. ${ }^{47}$ Neural cell adhesion molecule (NCAM) is involved in the regulation of neurotransmitter DA receptor $\mathrm{D}(2) \mathrm{R}$ transport and receptor-mediated signal transduction and behavior. ${ }^{48}$ DA acting on $\mathrm{D} 2$ receptor may regulate the structural plasticity of neurons and inhibit neurotransmission by changing the polysialylated form of NCAM expression. ${ }^{49}$ Maturation and NCAM-driven plasticity in dopaminergic brain areas can be affected by early life stress at different stages of ontogenesis and in a sex-specific manner. ${ }^{50}$ NCAM can mediate the survival of DA neurons through signaling pathways induced by mitogen-activated protein and ERK kinase, fibroblast growth factor receptor, protein kinase $\mathrm{A}$, and protein kinase $\mathrm{C} .{ }^{51} \mathrm{In}$ addition, the phagocytic inflammatory response of microglia leads to the complementmediated loss of dopaminergic neurons, leading to neurodegeneration. ${ }^{52}$ The early endocytic and endocytosis pathways play an important role in supporting rapid dopaminergic neurotransmission. ${ }^{53}$ Heptahelical G protein-coupled receptors (GPCRs) comprise the largest superfamily of signal transduction receptors, including adrenergic and dopaminergic receptors. Endocytic membrane transporter receptors to lysosomes play an important role in promoting down-regulation of GPCRs in some nerve cell types. Different GPCR endocytosis pathways may operate in parallel in the same cell, internalizing structurally homologous GPCR subtypes to different extents. ${ }^{54}$ Clarifying how GPCR signaling and membrane trafficking regulate specific subtypes of adrenergic and DA receptors could significantly advance the treatment of post-stroke depression. To summarize, DA system lesion plays a key role in the pathophysiology of post-ICH depression, and endocytosis, CAMs, and phagosome signaling may play an important role in this mechanism. 

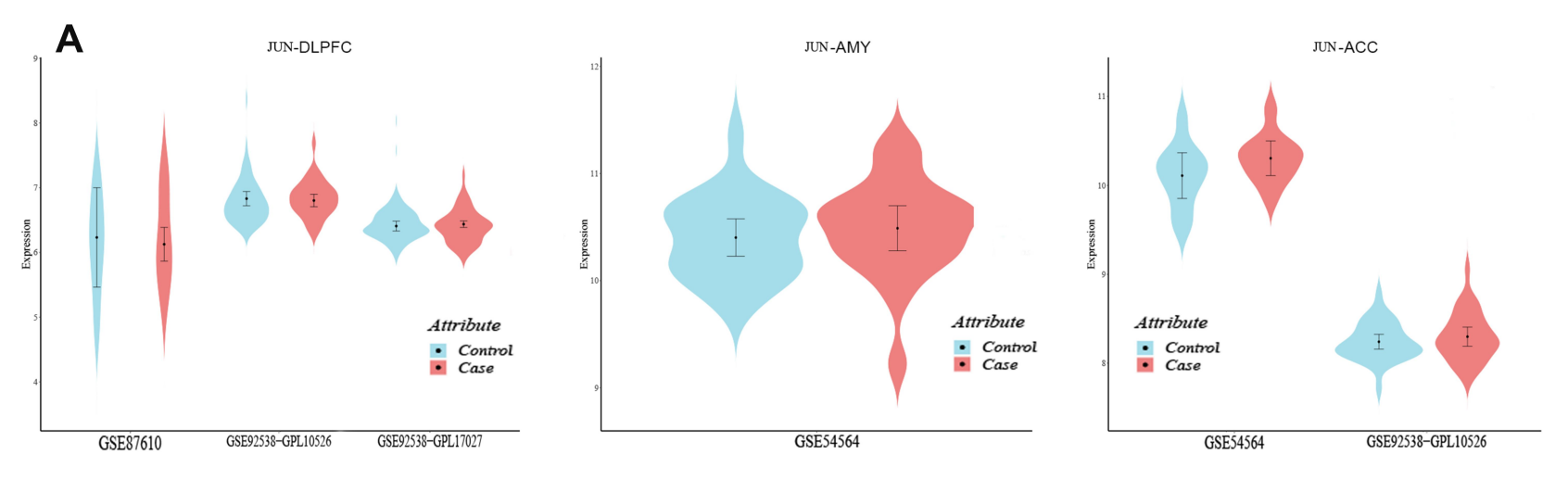

B
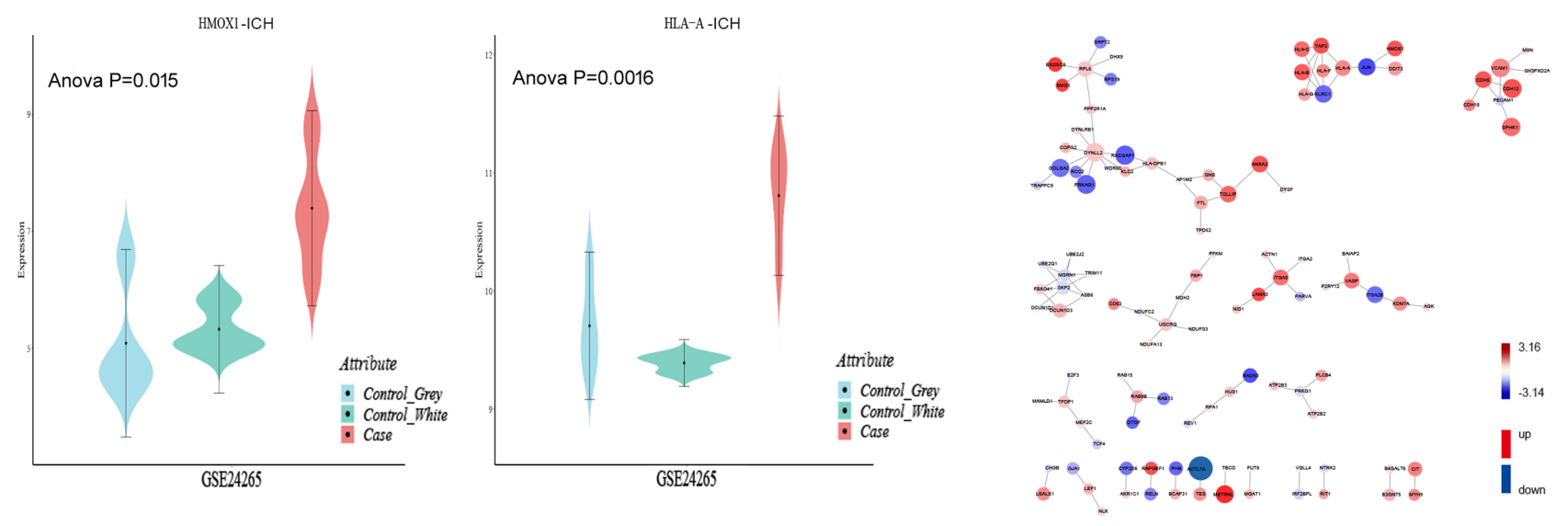

Figure 7 The hub bridge gene expression and the integrated regulatory network of bridge genes interactions. (A) Expression values of hub bridge genes in patients and healthy controls in different datasets. The $y$-axis is the standardized value of gene expression, and the $x$-axis indicates the dataset. (B) The integrated regulatory network of bridge genes interactions. The size of the node reflects the integrated driving force for the bridge genes. The shade of color represents the significance of the difference from blue (low) to red (high).

In addition, JUN, NCAM, and endocytosis play a role in 5-HT-related depression and antidepressant mechanisms. The human 5-HT(6) receptor [5-HT(6)R] interacts with Jun activation domain-binding protein-1 (Jab1). Jab1 provides a novel signal transduction pathway for 5-HT(6)R and may play an important role in 5-HT(6)R-mediated changes in the brain, including changes associated with depression. ${ }^{55}$ The G-allele of rs6295 is known to be associated with aspects of major depression, and the rs6295 G-/C-allelic variant is located in the promoter region of the human HTRla gene, which encodes the G-protein-coupled receptor for 5-HT. C-Jun activates the rs6295 G-allelic variant. ${ }^{56}$ NCAM L1 may serve as a biomarker of response to selective serotonin reuptake inhibitors (SSRIs). The close homolog of L1 (CHL1) is a CAM involved in the regulation of neuronal survival and growth. Downregulation of CHL1 in immune cells and brain tissue may be related to the immune pathogenesis of depression. ${ }^{57}$ The 5-HT1A receptor, a common GPCR associated with neuropsychiatric disorders such as depression, is an important drug target. The 5-HT1A receptor undergoes endocytosis mainly through the clathrin-mediated pathway and then circulates to the plasma membrane through the recycling endosome. The molecular mechanism of GPCR endocytosis may provide new insights into the potential mode of action of antidepressants acting through the serotonergic pathway. ${ }^{58}$

Although the present study provides new insights into the links between ICH and depression, it also contains several limitations. First, since brain specimens are very difficult to obtain, only 11 brain specimens of ICH were included in the present study. Second, we did not apply multiple testing correction in our statistical analyses. Third, our results are based on postmortem samples and so should be validated in vitro and in vivo, especially the 


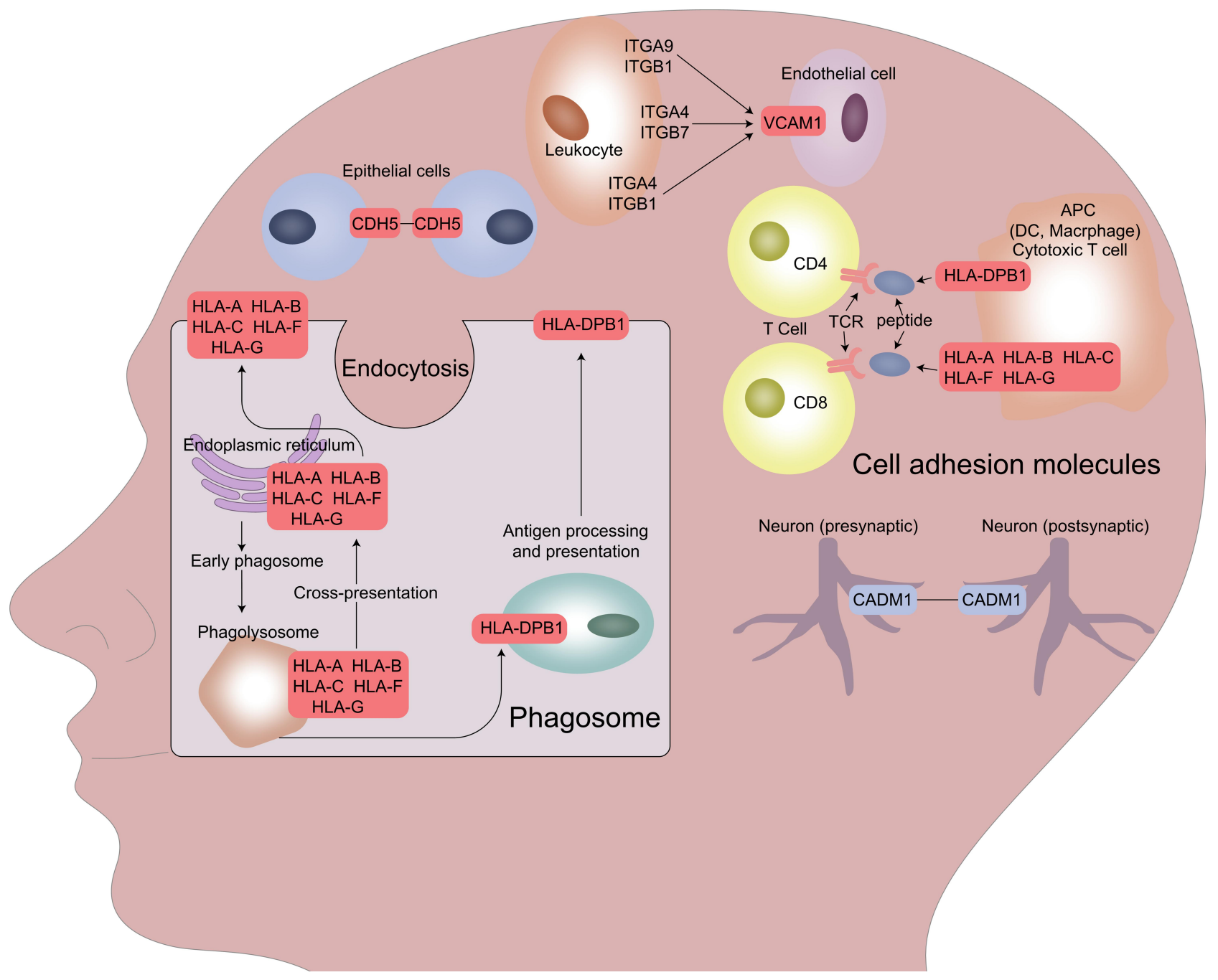

Figure 8 Proposed potential mechanisms of post-ICH depression. HLA-A, together with other hub bridge genes, regulates endocytosis, cell adhesion molecules, and phagosome pathways. The genes in the red and blue boxes are differentially expressed genes (DEGs): red indicates up-regulated genes; blue, down-regulated genes.

differential expression of hub bridge genes. Indeed, as our project was based purely on bioinformatics, its predictions of interactions between hub genes should be validated in molecular experiments.

Future research should explore the role of $H L A-A$, $H M O X 1, J U N$, endocytosis, CAMs, and phagosome signaling in models of post-ICH depression and in patients. Hopefully, this future work will clarify the underlying mechanism and highlight novel avenues for therapeutic intervention.r

\section{Conclusion}

We identified the hub bridge genes and signaling pathways related to post-ICH depression from a global, unbiased perspective, providing candidate molecules and functional pathways for further studies aimed at elucidating the pathogenesis of post-ICH depression.

\section{Funding}

This study received support from the National Natural Science Foundation of China (81860226), the Guangxi Natural Science Foundation (2018GXNSFAA294154), and the Guangxi Medical and Health Appropriate Technology Development and Application Project (S2018073).

\section{Disclosure}

The authors report no conflicts of interest in this work. 


\section{References}

1. Johnson CO, Nguyen M, Roth GA, et al. Global, regional, and national burden of stroke, 1990-2016: a systematic analysis for the Global Burden of Disease Study 2016. Lancet Neurol. 2019;18 (5):439-458. doi:10.1111/j.1460-9568.2007.05436.x

2. Wu S, Wu B, Liu M, et al. Stroke in China: advances and challenges in epidemiology, prevention, and management. Lancet Neurol. 2019;18(4):394-405. doi:10.1016/S1474-4422(18)30500-3

3. Jokinen H, Melkas S, Ylikoski R, et al. Post-stroke cognitive impairment is common even after successful clinical recovery. Eur J Neurol. 2015;22(9):1288-1294. doi:10.1111/ene.12743

4. Zhao F, Yue Y, Jiang H, Yuan Y. Shared genetic risk factors for depression and stroke. Prog Neuropsychopharmacol Biol Psychiatry. 2019;93:55-70. doi:10.1016/j.pnpbp.2019.03.003

5. Gilsanz P, Walter S, Tchetgen Tchetgen EJ, et al. Changes in depressive symptoms and incidence of first stroke among middle-aged and older US adults. J Am Heart Assoc. 2015;4(5). doi:10.1161/ JAHA.115.001923.

6. Stern-Nezer S, Eyngorn I, Mlynash M, et al. Depression one year after hemorrhagic stroke is associated with late worsening of outcomes. NeuroRehabilitation. 2017;41(1):179-187. doi:10.3233/ NRE-171470

7. Hackett ML, Pickles K. Part I: frequency of depression after stroke: an updated systematic review and meta-analysis of observational studies. Int J Stroke. 2014;9(8):1017-1025. doi:10.1111/ijs.12357

8. Flaherty ML, Haverbusch M, Sekar P, et al. Long-term mortality after intracerebral hemorrhage. Neurology. 2006;66(8):1182-1186. doi:10.1212/01.wnl.0000208400.08722.7c

9. Lioutas VA, Beiser AS, Aparicio HJ, et al. Assessment of incidence and risk factors of intracerebral hemorrhage among participants in the Framingham Heart Study between 1948 and 2016. JAMA Neurol. 2020;77:1252. doi:10.1001/jamaneurol.2020.1512

10. Fisher OS, Deng H, Liu D, et al. Structure and vascular function of MEKK3-cerebral cavernous malformations 2 complex. Nat Commun. 2015;6:7937. doi:10.1038/ncomms8937

11. Sun Y, Liu XL, Zhang D, et al. Platelet-derived exosomes affect the proliferation and migration of human umbilical vein endothelial cells via miR-126. Curr Vasc Pharmacol. 2019;17(4):379-387. doi:10.2174/1570161116666180313142139

12. Wang X, Hou Y, Deng K, Zhang Y, Wang DC, Ding J. Structural Insights into the Molecular Recognition between Cerebral Cavernous Malformation 2 and Mitogen-Activated Protein Kinase Kinase Kinase 3. Structure. 2015;23(6):1087-1096. doi:10.1016/j. str.2015.04.003

13. Wang Y, Pan L, Moens CB, Appel B. Notch3 establishes brain vascular integrity by regulating pericyte number. Development. 2014;141(2):307-317. doi:10.1242/dev.096107

14. Xing C, Wang X, Cheng C, et al. Neuronal production of lipocalin-2 as a help-me signal for glial activation. Stroke. 2014;45 (7):2085-2092. doi:10.1161/STROKEAHA.114.005733

15. Yang J, Wang Y, Zeng Z, et al. Smad4 deletion in blood vessel endothelial cells promotes ovarian cancer metastasis. Int $J$ Oncol. 2017;50(5):1693-1700. doi:10.3892/ijo.2017.3957

16. Zhou HJ, Tang T, Zhong JH, et al. Electroacupuncture improves recovery after hemorrhagic brain injury by inducing the expression of angiopoietin-1 and -2 in rats. BMC Complement Altern Med. 2014;14:127. doi:10.1186/1472-6882-14-127

17. Woo D, Falcone GJ, Devan WJ, et al. Meta-analysis of genome-wide association studies identifies 1q22 as a susceptibility locus for intracerebral hemorrhage. Am J Hum Genet. 2014;94(4):511-521. doi:10.1016/j.ajhg.2014.02.012

18. Koivunen RJ, Harno H, Tatlisumak T, Putaala J. Depression, anxiety, and cognitive functioning after intracerebral hemorrhage. Acta Neurol Scand. 2015;132(3):179-184. doi:10.1111/ane.12367
19. Wu Y, Wang $\mathrm{L}, \mathrm{Hu} \mathrm{K}$, et al. Mechanisms and therapeutic targets of depression after intracerebral hemorrhage. Front Psychiatry. 2018;9:682. doi:10.3389/fpsyt.2018.00682

20. Ritchie ME, Phipson B, Wu D, et al. limma powers differential expression analyses for RNA-sequencing and microarray studies. Nucleic Acids Res. 2015;43(7):e47. doi:10.1093/nar/gkv007

21. Yu G, Wang LG, Han Y, He QY. clusterProfiler: an R package for comparing biological themes among gene clusters. OMICS. 2012;16 (5):284-287. doi:10.1089/omi.2011.0118

22. Subramanian A, Tamayo P, Mootha VK, et al. Gene set enrichment analysis: a knowledge-based approach for interpreting genome-wide expression profiles. Proc Natl Acad Sci U S A. 2005;102 (43):15545-15550. doi:10.1073/pnas.0506580102

23. Liberzon A, Birger C, Thorvaldsdottir H, Ghandi M, Mesirov JP, Tamayo P. The Molecular Signatures Database (MSigDB) hallmark gene set collection. Cell Syst. 2015;1(6):417-425. doi:10.1016/j. cels.2015.12.004

24. Szklarczyk D, Morris JH, Cook H, et al. The STRING database in 2017: quality-controlled protein-protein association networks, made broadly accessible. Nucleic Acids Res. 2017;45(D1):D362-D368. doi:10.1093/nar/gkw937

25. Shannon P, Markiel A, Ozier O, et al. Cytoscape: a software environment for integrated models of biomolecular interaction networks. Genome Res. 2003;13(11):2498-2504. doi:10.1101/gr.1239303

26. Yu G, Li F, Qin Y, Bo X, Wu Y, Wang S. GOSemSim: an R package for measuring semantic similarity among GO terms and gene products. Bioinformatics. 2010;26(7):976-978. doi:10.1093/bioinformatics/btq064

27. Rahman MS, Yang J, Luan Y, et al. Attenuation of acute intracerebral hemorrhage-induced microglial activation and neuronal death mediated by the blockade of metabotropic glutamate receptor 5 in vivo. Neurochem Res. 2020;45(5):1230-1243. doi:10.1007/s11064020-03006-1

28. Wang W, Qin X, Wang R, et al. EZH2 is involved in vulnerability to neuroinflammation and depression-like behaviors induced by chronic stress in different aged mice. J Affect Disord. 2020;272:452-464. doi:10.1016/j.jad.2020.03.154

29. Wen H, Weymann KB, Wood L, Wang QM. Inflammatory signaling in post-stroke fatigue and depression. Eur Neurol. 2018;80(3-4):138-148. doi:10.1159/000494988

30. Nguyen VA, Carey LM, Giummarra L, et al. A pathway proteomic profile of ischemic stroke survivors reveals innate immune dysfunction in association with mild symptoms of depression - a pilot study. Front Neurol. 2016;7:85. doi:10.3389/fneur.2016.00085

31. Jiang JL, El Mansari M, Blier P. Triple reuptake inhibition of serotonin, norepinephrine, and dopamine increases the tonic activation of alpha2-adrenoceptors in the rat hippocampus and dopamine levels in the nucleus accumbens. Prog Neuropsychopharmacol Biol Psychiatry. 2020;103:109987. doi:10.1016/j.pnpbp.2020.109987

32. Zou XH, Sun LH, Yang W, Li BJ, Cui RJ. Potential role of insulin on the pathogenesis of depression. Cell Prolif. 2020;53(5):e12806. doi:10.1111/cpr.12806

33. Jin J, Bhatti DL, Lee KW, et al. Ahnak scaffolds p11/Anxa2 complex and L-type voltage-gated calcium channel and modulates depressive behavior. Mol Psychiatry. 2020;25(5):1035-1049. doi:10.1038/ s41380-019-0371-y

34. Weiss R, Bitton A, Ben Shimon M, et al. Annexin A2, autoimmunity, anxiety and depression. J Autoimmun. 2016;73:92-99. doi:10.1016/j. jaut.2016.06.011

35. Hedhli N, Falcone DJ, Huang B, et al. The annexin A2/S100A10 system in health and disease: emerging paradigms. $J$ Biomed Biotechnol. 2012;2012:406273. doi:10.1155/2012/406273

36. Sayad A, Akbari MT, Inoko H, et al. Association between human leucocyte antigen alleles and risk of stroke in Iranian population. Int J Immunogenet. 2019;46(3):179-191. doi:10.1111/iji.12421 
37. Glanville KP, Coleman JRI, Hanscombe KB, et al. Classical Human Leukocyte Antigen Alleles and C4 Haplotypes Are Not Significantly Associated With Depression. Biol Psychiatry. 2020;87(5):419-430. doi:10.1016/j.biopsych.2019.06.031

38. Majsky A, Dvorakova M, Zvolsky P. [Modifications of lymphocyte HL-A antigens as a consequence of therapy in patients with manic-depressive psychosis]. Folia Haematol Int Mag Klin Morphol Blutforsch. 1978;105(4):509-517. German.

39. Tran NQV, Nguyen AN, Takabe K, Yamagata Z, Miyake K. Pretreatment with amitriptyline causes epigenetic up-regulation of neuroprotection-associated genes and has anti-apoptotic effects in mouse neuronal cells. Neurotoxicol Teratol. 2017;62:1-12. doi:10.1016/j.ntt.2017.05.002

40. Yang Y, Dong B, Lu J, Wang G, Yu Y. Hemopexin reduces blood-brain barrier injury and protects synaptic plasticity in cerebral ischemic rats by promoting EPCs through the HO-1 pathway. Brain Res. 2018;1699:177-185. doi:10.1016/j.brainres.2018.08.008

41. Leclerc JL, Li C, Jean S, et al. Temporal and age-dependent effects of haptoglobin deletion on intracerebral hemorrhage-induced brain damage and neurobehavioral outcomes. Exp Neurol. 2019;317:22-33. doi:10.1016/j.expneurol.2019.01.011

42. Chen-Roetling J, Kamalapathy P, Cao Y, Song W, Schipper HM, Regan RF. Astrocyte heme oxygenase-1 reduces mortality and improves outcome after collagenase-induced intracerebral hemorrhage. Neurobiol Dis. 2017;102:140-146. doi:10.1016/j.nbd.2017.03.008

43. Zheng J, Dai Q, Han K, et al. JNK-IN-8, a c-Jun N-terminal kinase inhibitor, improves functional recovery through suppressing neuroinflammation in ischemic stroke. $J$ Cell Physiol. 2020;235 (3):2792-2799. doi:10.1002/jcp.29183

44. Zhang J, Lin $\mathrm{W}$, Tang $\mathrm{M}$, et al. Inhibition of JNK ameliorates depressive-like behaviors and reduces the activation of pro-inflammatory cytokines and the phosphorylation of glucocorticoid receptors at serine 246 induced by neuroinflammation. Psychoneuroendocrinology. 2020;113:104580. doi:10.1016/j.psyneuen.2019.104580

45. Lu H, Ning X, Tao X, et al. MEKK1 associated with neuronal apoptosis following intracerebral hemorrhage. Neurochem Res. 2016;41(12):3308-3321. doi:10.1007/s11064-016-2063-1

46. Yang Y, Zhang K, Zhong J, et al. Stably maintained microtubules protect dopamine neurons and alleviate depression-like behavior after intracerebral hemorrhage. Sci Rep. 2018;8(1):12647. doi:10.1038/ s41598-018-31056-7

47. Sharaf A, Bock HH, Spittau B, Bouche E, Krieglstein K. ApoER2 and VLDLr are required for mediating reelin signalling pathway for normal migration and positioning of mesencephalic dopaminergic neurons. PLoS One. 2013;8(8):e71091. doi:10.1371/journal.pone.0071091
48. Xiao MF, Xu JC, Tereshchenko Y, Novak D, Schachner M, Kleene R. Neural cell adhesion molecule modulates dopaminergic signaling and behavior by regulating dopamine $\mathrm{D} 2$ receptor internalization. $J$ Neurosci. 2009;29(47):14752-14763. doi:10.1523/JNEUROSCI.4860-09.2009

49. Castillo-Gomez E, Gomez-Climent MA, Varea E, et al. Dopamine acting through D2 receptors modulates the expression of PSA-NCAM, a molecule related to neuronal structural plasticity, in the medial prefrontal cortex of adult rats. Exp Neurol. 2008;214 (1):97-111. doi:10.1016/j.expneurol.2008.07.018

50. Chocyk A, Dudys D, Przyborowska A, Mackowiak M, Wedzony K. Impact of maternal separation on neural cell adhesion molecules expression in dopaminergic brain regions of juvenile, adolescent and adult rats. Pharmacol Rep. 2010;62(6):1218-1224. doi:10.1016/ S1734-1140(10)70385-6

51. Ditlevsen DK, Berezin V, Bock E. Signalling pathways underlying neural cell adhesion molecule-mediated survival of dopaminergic neurons. Eur J Neurosci. 2007;25(6):1678-1684.

52. Bodea LG, Wang Y, Linnartz-Gerlach B, et al. Neurodegeneration by activation of the microglial complement-phagosome pathway. $J \quad$ Neurosci. 2014;34(25):8546-8556. doi:10.1523/ JNEUROSCI.5002-13.2014

53. Kotowski SJ, Hopf FW, Seif T, Bonci A, von Zastrow M. Endocytosis promotes rapid dopaminergic signaling. Neuron. 2011;71(2):278-290. doi:10.1016/j.neuron.2011.05.036

54. von Zastrow M. Endocytosis and downregulation of G protein-coupled receptors. Parkinsonism Relat Disord. 2001;7 (3):265-271. doi:10.1016/S1353-8020(00)00069-9

55. Yun HM, Baik JH, Kang I, Jin C, Rhim H. Physical interaction of Jab1 with human serotonin 6 G-protein-coupled receptor and their possible roles in cell survival. $J$ Biol Chem. 2010;285 (13):10016-10029. doi:10.1074/jbc.M109.068759

56. Pernhorst K, van Loo KM, von Lehe M, et al. Rs6295 promoter variants of the serotonin type $1 \mathrm{~A}$ receptor are differentially activated by c-Jun in vitro and correlate to transcript levels in human epileptic brain tissue. Brain Res. 2013;1499:136-144. doi:10.1016/j. brainres.2012.12.045

57. Yang CR, Ning L, Zhou FH, et al. Downregulation of adhesion molecule CHL1 in B cells but not $\mathrm{T}$ cells of patients with major depression and in the brain of mice with chronic stress. Neurotox Res. 2020;38(4):914-928. doi:10.1007/s12640-020-00234-9

58. Kumar GA, Sarkar P, Jafurulla M, et al. Exploring endocytosis and intracellular trafficking of the human Serotonin1A receptor. Biochemistry. 2019;58(22):2628-2641. doi:10.1021/acs. biochem.9b00033
International Journal of General Medicine

\section{Publish your work in this journal}

The International Journal of General Medicine is an international, peer-reviewed open-access journal that focuses on general and internal medicine, pathogenesis, epidemiology, diagnosis, monitoring and treatment protocols. The journal is characterized by the rapid reporting of reviews, original research and clinical studies across all disease areas. The manuscript management system is completely online and includes a very quick and fair peer-review system, which is all easy to use. Visit http://www.dovepress.com/ testimonials.php to read real quotes from published authors. 